\title{
Developing Quantum Dot Phosphor-Based Light-Emitting Diodes for Aviation Lighting Applications
}

\author{
Fengbing Wu, ${ }^{1}$ Dawei Zhang,, Shuzhen Shang, ${ }^{1}$ Yiming Zhu, ${ }^{1}$ \\ Songlin Zhuang, ${ }^{1}$ and Jian $\mathrm{Xu}^{1,2}$ \\ ${ }^{1}$ Engineering Research Center of Optical Instrument and System, University of Shanghai for Science and Technology, \\ No. 516 JunGong Road, Shanghai 200093, China \\ ${ }^{2}$ Department of Engineering Science and Mechanics, Pennsylvania State University, University Park, PA 16802, USA
}

Correspondence should be addressed to Jian Xu, jianxu@engr.psu.edu

Received 10 October 2011; Revised 4 January 2012; Accepted 4 January 2012

Academic Editor: Sylvain G. Cloutier

Copyright (C) 2012 Fengbing Wu et al. This is an open access article distributed under the Creative Commons Attribution License, which permits unrestricted use, distribution, and reproduction in any medium, provided the original work is properly cited.

\begin{abstract}
We have investigated the feasibility of employing quantum dot (QD) phosphor-based light-emitting diodes (LEDs) in aviation applications that request Night Vision Imaging Systems (NVIS) compliance. Our studies suggest that the emerging QD phosphorbased LED technology could potentially be superior to conventional aviation lighting technology by virtue of the marriage of tight spectral control and broad wavelength tunability. This largely arises from the fact that the optical properties of semiconductor nanocrystal QDs can be tailored by varying the nanocrystal size without any compositional changes. It is envisioned that the QD phosphor-based LEDs hold great potentials in cockpit illumination, back light sources of monitor screens, as well as the LED indicator lights of aviation panels.
\end{abstract}

\section{Introduction}

The essence of Night Vision Imaging Systems (NVIS) compliance in aviation lighting technology is to ensure that the pilots can successfully operate avionic systems when wearing NVIS goggles under cockpit illumination conditions. This paper reports the feasibility of employing quantum dot (QD) phosphor-based light-emitting diodes (LEDs) in aviation applications that request Night Vision Imaging Systems (NVIS) compliance.

NVIS technology refers to the application of photoelectron systems that perform image information transformation, enhancement, processing, display, and other physical processes. At the heart of such a system is an image intensifier which enhances the infrared signals under low-levellight (LLL) conditions. The third generation NVIS uses Gallium Arsenide photocathode image enhancement tube. The maximum response falls in the near-infrared wavelength. A typical product is the AN/AVS-6 LLL night vision system [1]. The system needs to install filters to reduce the influence of visible light. The filters can be divided into two generic types, that is, Class A with the maximum response wavelengths between $625 \sim 930 \mathrm{~nm}$ and Class B with the maximum response wavelengths between $665 \sim 930 \mathrm{~nm}$ [2].

While pilots rely on the visible illumination to read aviation panels and display screens in cockpits, the light sources also produce some radiation in near-infrared (IR) regime [3]. Although the influence of visible light to AN/AVS- 6 has been greatly reduced by employing Class A or B filters, the infrared radiation from the cockpit light sources often overlaps with the NVIS spectral response. The near-IR radiance entering the aperture of pilots' NVIS goggles will severely compromise the use of NVIS, making the goggles glaring, and sometimes even burning the image intensifier tube. It is therefore necessary to lower the degree of near-infrared radiation significantly using NVIS-compatible lighting technology.

The aviation standard usually uses a set of parameters to characterize the cockpit lighting for NVIS compatibility, that is, NVIS radiance, color limits, and visible light transmittance [3]. Among them, the NVIS radiance and color limits are the most critical indicators. 
The NVIS radiance can always be calculated by the following formula:

NVIS radiance $\left(\mathrm{NR}_{\mathrm{A} \text { or } \mathrm{B}}\right)$ at specified luminance

$$
=S \int_{450}^{930} G_{\mathrm{A} \text { or B }}(\lambda) N(\lambda) d \lambda,
$$

where $G_{\mathrm{A}}(\lambda)=$ relative NVIS response of Class A equipment, $G_{\mathrm{B}}(\lambda)=$ relative NVIS response of Class B equipment, $N(\lambda)=$ spectral radiance of lighting component $\left(W / \mathrm{sr} \times \mathrm{cm}^{2} \times \mathrm{nm}\right)$, $S=$ scaling factor.

The basic requirements of NVIS radiance is $-1.0 \leq \mathrm{NR} \leq$ $1.7 E-10$.

The colors and color limits are determined by the following formula:

$$
\left(u^{\prime}-u_{1}^{\prime}\right)^{2}+\left(v^{\prime}-v_{1}^{\prime}\right)^{2} \leq r^{2},
$$

where $u^{\prime} \& v^{\prime}=1976$ UCS chromaticity coordinates of the test article, $u_{1}^{\prime} \& v_{1}^{\prime}=1976$ UCS chromaticity coordinates of the center point of the specified color area, $r=$ radius of the allowable circular area on the 1976 UCS chromaticity diagram for the specified color.

These lighting colors and limits are designated as "NVIS GREEN A," "NVIS GREEN B," "NVIS YELLOW," "NVIS RED," and "NVIS WHITE" [3], and NVIS white is the most common color of the light sources in aviation applications.

Colloidal compound QDs have recently been introduced to the white LED technology as a new family of phosphor materials with many superior properties [4-7]. Due to strong quantum confinement, semiconductor QDs, such as core/ shell CdSe/(Zn, Cd)S QDs, are characterized by sharp exciton absorption features, extremely high luminescence efficiency ( $\sim 90-95 \%)$, and size-tunable emission color spanning the entire visible spectrum [8]. QDs of the same chemical composition and different size can therefore be employed to provide multiple spectral components in white LEDs output, with improved color qualities and aging performance [9]. Perhaps the most significant potential of QD phosphors lies in the fact that the extremely narrow emission band of monodisperse QDs populations (FWHM $\sim 15-30 \mathrm{~nm}$ ) [10]. II-VI compound-based QD-LEDs have produced color-saturated emissions of much higher spectral purities than those of liquid crystal displays (LCDs) and organic light emitting diodes (OLEDs) and even 30\% greater than bulky cathode ray tubes (CRTs) that are still favored for their excellent color rendition. It is therefore possible to fine tailor the color rendering index (CRI), color temperature as well as the NVIS radiance of white QD phosphor-based LEDs in order to meet the strict spectral requirements in a variety of special LED applications.

In the present work, white emission from QD phosphorbased LEDs is achieved by depositing a layer of solutionprocessed QD film, composed of multi-color $\mathrm{Cd}(\mathrm{S}, \mathrm{Se}) /(\mathrm{Zn}$, Cd)S quantum dots, on top of the emissive surfaces of blueemitting nitride LEDs [11-15]. Unlike traditional fluorescent lamps where only one type of UV-sensitive broadband phosphor is employed, QD phosphor-based LEDs produce white light by mixing two compensating colors (blue and yellow/orange) or several primary colors (red, yellow, green, and blue). By varying the nanocrystal sizes or adjusting the compound composition, the luminescence spectrum of the monodisperse quantum dots can cover the entire visible light spectrum. It is therefore possible to obtain spectrally-adjustable white light QD phosphor-based LEDs by integrating multicolor QD phosphors in the LED device. Finally, by engineering the surfactant molecules of the nanocrystals, it is possible to further enhance the fluorescence quantum yield and make the QDs more photochemical stable, leading to enhanced efficiency and output luminance of white QD phosphor-based LEDs $[16,17]$.

\section{Methods}

When QD phosphor-based LEDs are used as the light sources in the aircraft cockpit, it is important to make the LED output compatible with NVIS requirements. This sets rigorous limits on the emission spectra of the LEDs, especially on the reddish side of the band. For traditional white fluorescent lighting systems employed in the aircraft cockpit, the NVIS Radiance Class B (NRB) is measured $\sim 1 \times 10^{-6}(0.1 \mathrm{ft})$, which is much higher than the specified threshold $(2.2 E-9)$ of NVIS white. The high NRB of the traditional lighting systems arises from the broadband emission of the UV-sensitive phosphors and can only be reduced via filters [18-22]. On the other hand, the NRB of the existing commercial white LEDs falls in the range of $1 \times 10^{-7} \sim 1 \times 10^{-8}$, which is still one or two orders of magnitude lower than the NVIS threshold. This arises from the fact that the downconversion of blue radiation to yellow/green and red in conventional LEDs was accomplished with $\mathrm{Y}_{3} \mathrm{Al}_{5} \mathrm{O}_{12}: \mathrm{Ce}^{3+}$ and $\mathrm{Eu}^{2+}$ doped nitridosilicates phosphors coated on top of the InGaN QW LEDs for color mixing in the white output. While the luminescence of the blue-sensitive $\mathrm{Y}_{3} \mathrm{Al}_{5} \mathrm{O}_{12}: \mathrm{Ce}^{3+}$ and $\mathrm{Eu}^{2+}$ doped nitridosilicates phosphors exhibits spectrally narrower band than the UV-sensitive phosphors in fluorescence lamps, there is still an appreciable near-IR portion $(\lambda \geq 650 \mathrm{~nm})$ in the luminescence spectrum of the blue-sensitive phosphors. Hence it is still necessary to implement filtering techniques with conventional white LEDs in order to meet the NVIS requirement.

By selecting the QD size, it is possible to precisely control the residual emission of the white light QD phosphor-based LEDs in near-IR regime. Hence it is feasible to fabricate high-luminous-efficiency white QD-LED chips that are compatible with NVIS without any filters. The spectral overlap between the output of QD phosphor-based LEDs and that of the night vision systems can be minimized. These QD phosphor-based LEDs hold great potentials in cockpit illumination, back light sources of monitor screens, as well as the LEDs indicator lights of aviation panels.

2.1. Calculation. In the present study, the NRB of a QD phosphor-based LED was calculated to illustrate the NVIScompatibility of QD phosphor-based LEDs. In this device, the blue emission of $450 \mathrm{~nm}$-peak wavelength of an InGaN QW LED is mixed with the yellow-orange luminescence of CdSe/CdS/ZnS core-shell QDs to produce "complimentary white" in the QD phosphor-based LED output. Figure 1(a) 


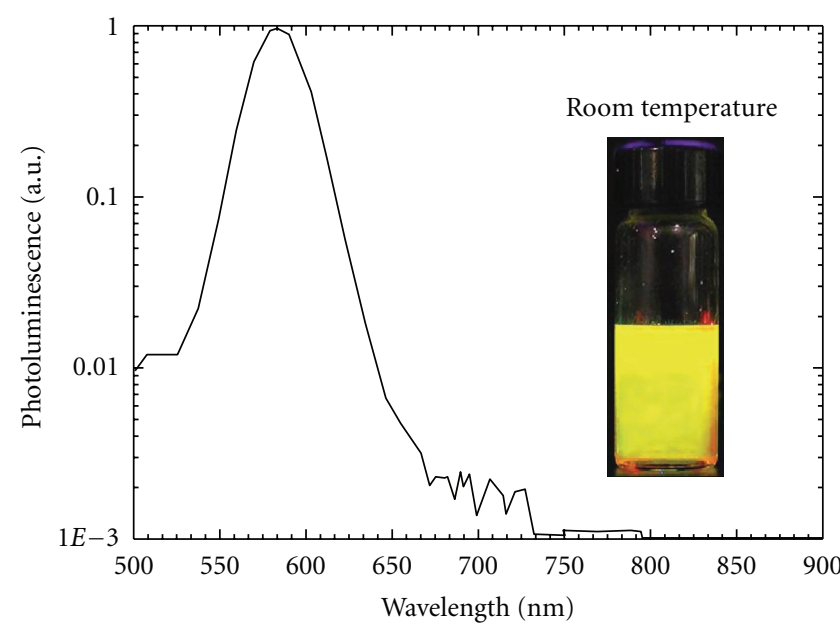

(a)

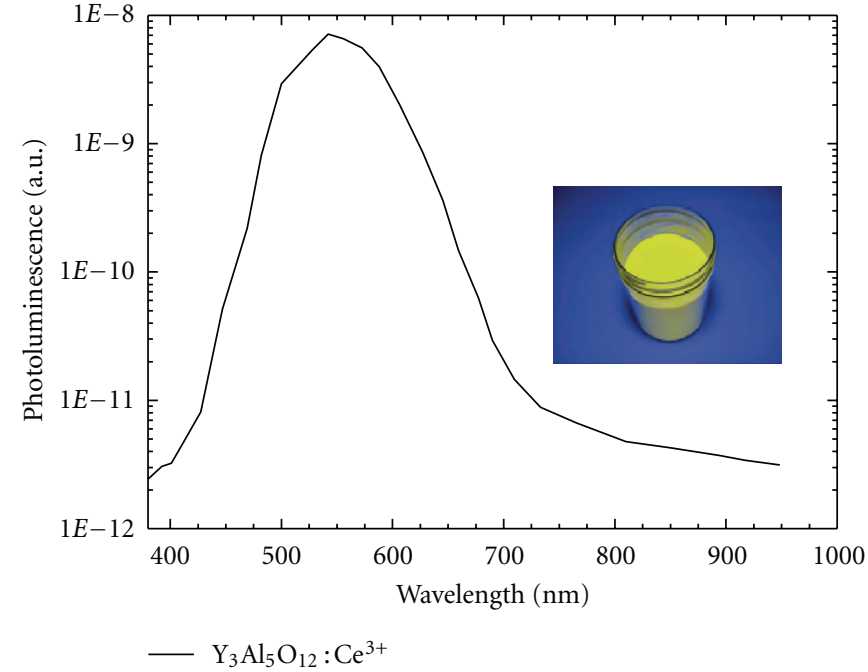

(b)

Figure 1: (a) The luminescence spectrum of yellow CdSe/CdS/ZnS core-shell QDs that is used in the calculation. (b) The luminescence spectra of $\mathrm{Y}_{3} \mathrm{Al}_{5} \mathrm{O}_{12}: \mathrm{Ce}^{3+}$ phosphor that is used in the calculation.

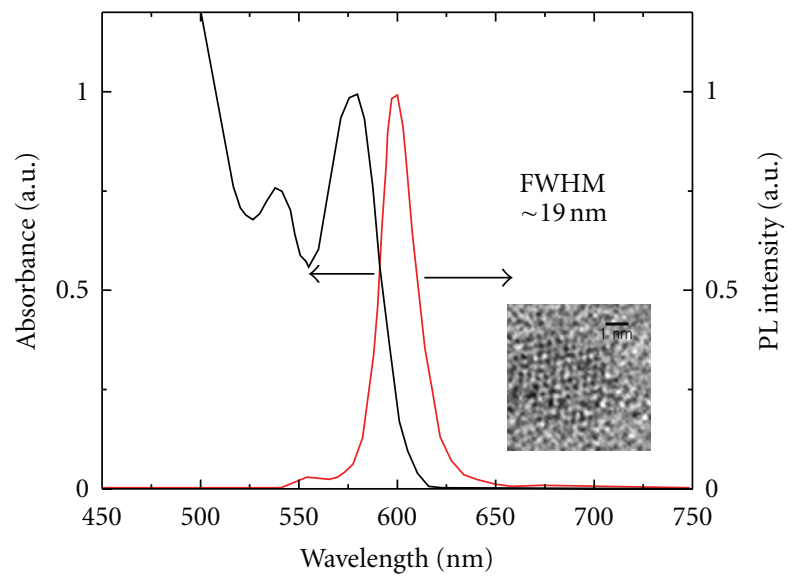

FIgure 2: Absorption and luminescence spectra of orange-red-emitting CdSe/CdS/ZnS core-shell QDs used in the proof-of-concept experiment. The inset shows the HRTEM image of an individual QD.

shows the luminescence spectrum of the QDs employed in the calculation. For the sake of comparison, the fluorescence spectrum of $\mathrm{Y}_{3} \mathrm{Al}_{5} \mathrm{O}_{12}: \mathrm{Ce}^{3+}$ phosphor is also plotted in Figure 1(b). Calculation using (1) shows that the NVIS radiance for Class B detection systems (NRB) of the QD phosphor-based LEDs can be as low as $4 E-10$ at $0.1 \mathrm{ft}$ brightness, which is far below the threshold radiance for Night Vision Imaging Systems (NVIS). Even at a high brightness ( $0.5 \mathrm{ft})$, the NRB $(2.0 E-9)$ of QD phosphor-based LEDs still complies with the NVIS requirement. In comparison, the NRB of an LED with $\mathrm{Y}_{3} \mathrm{Al}_{5} \mathrm{O}_{12}: \mathrm{Ce}^{3+}$ phosphor was calculated to be $\sim 6 \times 10^{-8}$ at $0.1 \mathrm{ft}$ brightness.

2.2. Experiments. In a proof-of-concept experiment, we have fabricated QD phosphor-based LEDs by mist-depositing orange-emitting CdSe/CdS/ZnS core-shell QDs over the emissive surface of InGaN QW LEDs [23]. The QDs employed in our study are orange-red CdSe/CdS/ZnS core-shell structures, which were synthesized following the method developed by Dr. Peng's group (CdSe-(Cd, Zn)S) [24]. Nearly monodisperse QDs were produced and stored in toluene solutions. Transmission electron microscopy was conducted to determine the lattice structure and dimensions of the QDs as shown in the inset of Figure 2. Nearly identical spherical crystalline particles are observed. The particles in the high resolution TEM micrographs show well-resolved lattice fringes with a measured lattice spacing similar to the bulk CdSe structure. The high-quality of the QDs was also manifested by the sharp exciton absorption features and narrow FWHM bandwidth of photoluminescence $(\sim 19 \mathrm{~nm})$ of the QDs (Figure 2).

The QDs were dispersed in toluene solution for mist deposition, and the thickness of the QDs was precisely 


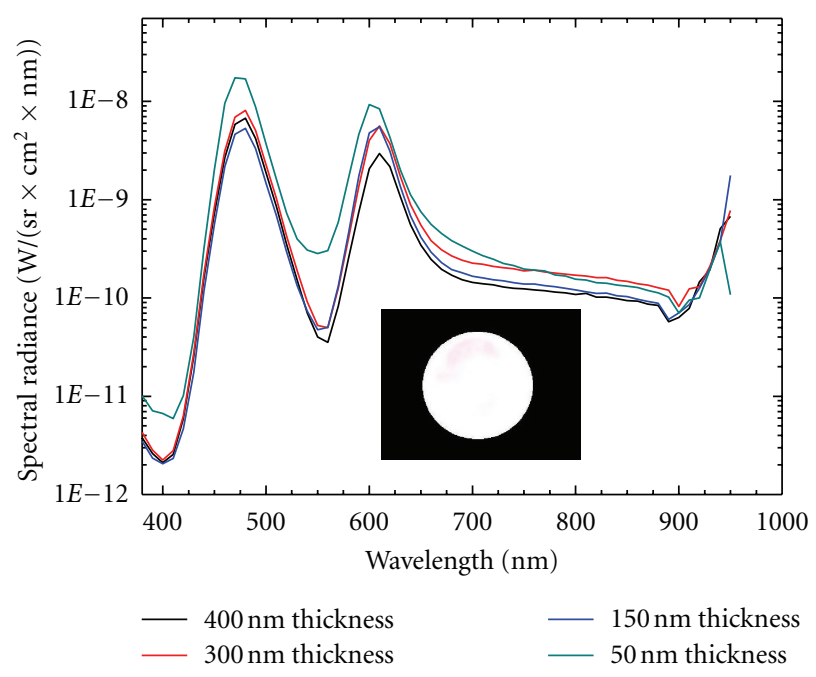

(a)

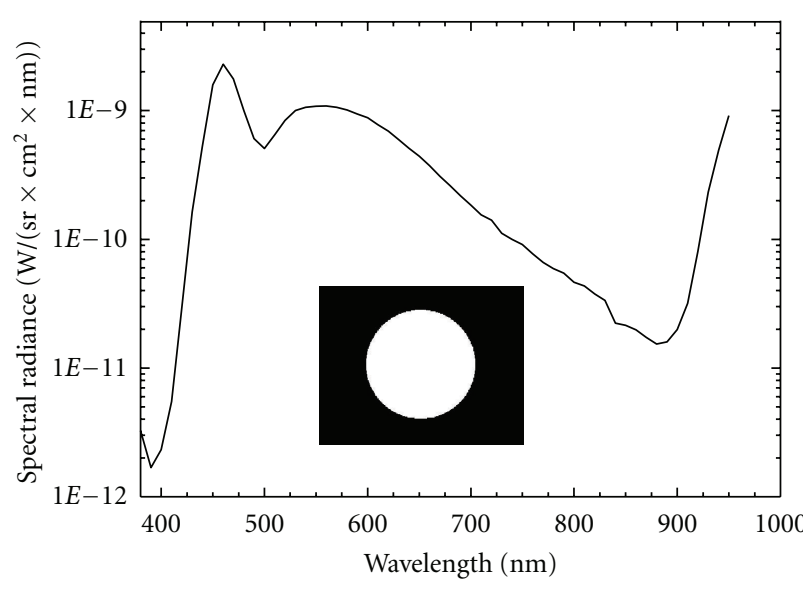

(b)

FIGURE 3: (a) The output spectra of QD phosphor-based LEDs with different QD film thicknesses. The inset shows a portion of the emissive surface of the white QD phosphor-based LED. (b) The output spectrum of a commercial LED. The inset shows a portion of the white emissive surface of the commercial LED.

TABLE 1: The NVIS radiance of a QD-LED and a commercial white LED.

\begin{tabular}{|c|c|c|c|}
\hline Model & $\begin{array}{c}\text { UCS } 1976 \text { chormaticity } \\
\text { coordinates }\end{array}$ & $\begin{array}{c}\text { Tested NVIS } \\
\left(W /\left(\mathrm{sr} \times \mathrm{cm}^{2} \times \mathrm{nm}\right)\right)\end{array}$ & $\begin{array}{c}\text { Specified NVIS } \\
\left(W /\left(\mathrm{sr} \times \mathrm{cm}^{2} \times \mathrm{nm}\right)\right)\end{array}$ \\
\hline QD-LEDs & $u^{\prime}=0.2315$ & \multirow{2}{*}{$\mathrm{NRB}=1.652 E-08$} & \multirow{8}{*}{$\mathrm{NRB} \leq 1.0 E-09$} \\
\hline Thickness $\sim 400 \mathrm{~nm}$ & $v^{\prime}=0.3815$ & & \\
\hline QD-LEDs & $u^{\prime}=0.2665$ & \multirow{2}{*}{$\mathrm{NRB}=1.673 E-08$} & \\
\hline Thickness $\sim 300 \mathrm{~nm}$ & $v^{\prime}=0.4089$ & & \\
\hline QD-LEDs & $u^{\prime}=0.2979$ & \multirow{2}{*}{$\mathrm{NRB}=1.335 E-08$} & \\
\hline Thickness $\sim 150 \mathrm{~nm}$ & $v^{\prime}=0.4368$ & & \\
\hline QD-LEDs & $u^{\prime}=0.2481$ & \multirow{2}{*}{$\mathrm{NRB}=9.420 E-09$} & \\
\hline Thickness $\sim 50 \mathrm{~nm}$ & $v^{\prime}=0.3918$ & & \\
\hline $\begin{array}{l}\text { Commercial } \\
\text { White LED }\end{array}$ & $u^{\prime}=0.1950$ & \multirow[t]{2}{*}{$\mathrm{NRB}=1.300 E-07$} & \multirow[t]{2}{*}{$\mathrm{NRB} \leq 1.0 E-09$} \\
\hline (LL-HP70MWG) & $v^{\prime}=0.4626$ & & \\
\hline
\end{tabular}

controlled by varying the deposition time, the flux of the carrier gas, and the concentration of the QDs solution. A deposition rate of $\sim 100 \mathrm{~nm} / \mathrm{min}$ for the formation of QDs films over the emissive surface of nitride LEDs is used in the present work. In processing white QD-LEDs, a type of InGaN-QW-based blue chips was used with a peak emission at $\lambda \sim 469 \mathrm{~nm}$, and a layer of orange-red QDs was deposited as the phosphor to produce complimentary white output. For the measurement, a Night Vision Display Test and Measurement System (OL 770-NVS, Gooch \& Housego) was utilized to characterize the output spectra and to determine the NVIS radiance of the QD phosphor-based LED devices under study. Figure 3(a) plots the output spectra of QD phosphor-based LEDs configured with $50 \mathrm{~nm}, 150 \mathrm{~nm}$, $300 \mathrm{~nm}$, and $400 \mathrm{~nm}$-thick QD phosphor films, respectively. The measured NVIS radiance was summarized in Table 1.
For comparison, we also measured the spectral response and NVIS radiance of a commercial white LED (Lucky Light LLHP70MWG) that use ceramic phosphor $\left(\mathrm{Y}_{3} \mathrm{Al}_{5} \mathrm{O}_{12}: \mathrm{Ce}^{3+}\right)$, which have also been included in Figure 3(b) and Table 1.

\section{Results and Discussion}

It is evident from our measurement results that the NRB of the commercial white LEDs falls in the range of $10^{-7}$, which is significantly off the NVIS specification without filters. On the other hand, the NVIS radiance of QD phosphor-based LEDs was found as low as 9.420E - 09 for a QD thickness of $50 \mathrm{~nm}$, which is more than an order of magnitude lower than that of commercial white light LEDs $\left(1.3 \times 10^{-7}\right)$. While it is still slightly higher than the NVIS requirement, it is envisioned that NRB of QD phosphor-based LEDs can be further 
reduced by blue shifting the luminescence wavelength of the deposited QD phosphors from orange-red into yelloworange or even yellow regime. As the spectral maxima of the QDs are shifted further away from the near-IR regime, there will be reduced overlap between the QD-LED emission and NVIS spectral response, and hence the reduced NRB value. Our measurement results also suggest the NVIS radiance increases along with the increasing thickness of the QD film. It is suspected that reabsorption-induced red shift of QD emission becomes more serious in thicker QD films, which accounts for the observed increase in NVIS radiance. Further investigation is currently underway to verify this hypothesis.

\section{Conclusions}

In summary, our calculation and proof-of-concept experiment suggest that white QD phosphor-based LEDs can be tailored to exhibit higher compatibility with NVIS than commercial LEDs. It is therefore envisioned that the QD phosphor-based LEDs hold great potentials in cockpit illumination, back light sources of monitor screens, as well as the LED indicator lights of aviation panels.

\section{Acknowledgments}

The work at University of Shanghai for Science and Technology is supported by NSFC under Grant 61078007, Shanghai Municipal Education Commission and Shanghai Education Development Foundation under Shu Guang Project 10SG46, Science and Technology Commission of Shanghai Municipality under Grants 11530502200 and 1052nm07100, and Program for New Century Excellent Talents in University. The work at the Penn State University is being supported by the National Science Foundation under Grants CMMI0729263 and ECCS 0824186.

\section{References}

[1] P. Wan-cong, Foreign Military Low-Light-Level (LLL) Night Vision Equipment, Weapon Industry Press, Beijing, China, 1991.

[2] Armament Standard Press of Commission of Science Technology and Industry for National Defense, GJB1394-92 Aircraft NVIS-Compatible Interior Lighting System, Armament Standard Press of Commission of Science Technology and Industry for National Defense, Beijing, China, 1994.

[3] Department of Defense, MIL-STD-3009 Lighting, Aircraft, Night Vision Imaging System (NVIS) Compatible, Department of Defense, Washington, DC, USA, 2001.

[4] S. V. Kershaw, M. Harrison, A. L. Rogach, and A. Kornowski, "Development of IR-emitting colloidal II-VI quantum-dot materials," IEEE Journal on Selected Topics in Quantum Electronics, vol. 6, no. 3, pp. 534-543, 2000.

[5] S. Coe-Sullivan, W.-K. Woo, J. S. Steckel, M. Bawendi, and V. Bulovi, "Tuning the performance of hybrid organic/inorganic quantum dot light-emitting devices," Organic Electronics, vol. 4, no. 2-3, pp. 123-130, 2003.

[6] J. S. Steckel, P. Snee, S. Coe-Sullivan et al., "Color-saturated green-emitting QD-LEDs," Angewandte Chemie-International Edition, vol. 45, no. 35, pp. 5796-5799, 2006.
[7] J. S. Steckel, J. P. Zimmer, S. Coe-Sullivan, N. E. Stott, V. Bulović, and M. G. Bawendi, "Blue luminescence from (CdS)ZnS core-shell nanocrystals," Angewandte Chemie-International Edition, vol. 43, no. 16, pp. 2154-2158, 2004.

[8] Q. Sun, Y. A. Wang, L. S. Li et al., "Bright, multicoloured lightemitting diodes based on quantum dots," Nature Photonics, vol. 1, no. 12, pp. 717-722, 2007.

[9] H. S. Chen, C. K. Hsu, and H. Y. Hong, "InGaN-CdSe-ZnSe quantum dots white LEDs," IEEE Photonics Technology Letters, vol. 18, no. 1, pp. 193-195, 2006.

[10] S. Chaudhary, M. Ozkan, and W. C. W. Chan, "Trilayer hybrid polymer-quantum dot light-emitting diodes," Applied Physics Letters, vol. 84, no. 15, pp. 2925-2927, 2004.

[11] M. Achermann, M. A. Petruska, D. D. Koleske, M. H. Crawford, and V. I. Klimov, "Nanocrystal-based light-emitting diodes utilizing high-efficiency nonradiative energy transfer for color conversion," Nano Letters, vol. 6, no. 7, pp. 13961400, 2006.

[12] M. C. Schlamp, X. Peng, and A. P. Alivisatos, "Improved efficiencies in light emitting diodes made with $\mathrm{CdSe}(\mathrm{CdS})$ core/ shell type nanocrystals and a semiconducting polymer," Journal of Applied Physics, vol. 82, no. 11, pp. 5837-5842, 1997.

[13] B. O. Dabbousi, M. G. Bawendi, O. Onitsuka, and M. F. Rubner, "Electroluminescence from CdSe quantum-dot/polymer composites," Applied Physics Letters, vol. 66, pp. 1316-1318, 1995.

[14] S. Coe, W.-K. Woo, M. Bawendi, and V. Bulović, "Electroluminescence from single monolayers of nanocrystals in molecular organic devices," Nature, vol. 420, no. 6917, pp. 800-803, 2002.

[15] S. Chaudhary, M. Ozkan, and W. C. W. Chan, "Trilayer hybrid polymer-quantum dot light-emitting diodes," Applied Physics Letters, vol. 84, no. 15, pp. 2925-2927, 2004.

[16] H. Yang, P. H. Holloway, and B. B. Ratna, "Photoluminescent and electroluminescent properties of $\mathrm{Mn}$-doped $\mathrm{ZnS}$ nanocrystals," Journal of Applied Physics, vol. 93, no. 1, pp. 586-592, 2003.

[17] C. B. Murray, C. R. Kagan, and M. G. Bawendi, "Synthesis and characterization of monodisperse nanocrystals and closepacked nanocrystal assemblies," Annual Review of Materials Science, vol. 30, pp. 545-610, 2000.

[18] V. I. Klimov, Semiconductor and Metal Nanocrystals Synthesis and Electronic and Optical Properties, Marcel Dekker, New York, NY, USA, 1st edition, 2004.

[19] Y. Xuan, D. Pan, N. Zhao, X. Ji, and D. Ma, "White electroluminescence from a poly(N-vinylcarbazole) layer doped with CdSe/CdS core-shell quantum dots," Nanotechnology, vol. 17, no. 19, pp. 4966-4969, 2006.

[20] G. Moeller and S. Coe-Sullivan, "Quantum-dot light-emitting devices for displays," Information Display, vol. 22, no. 2, pp. 2-6, 2006.

[21] P. O. Anikeeva, J. E. Halpert, M. G. Bawendi, and V. Bulović, "Electroluminescence from a mixed red-green-blue colloidal quantum dot monolayer," Nano Letters, vol. 7, no. 8, pp. 21962200, 2007.

[22] R. D. New, R. L. Burgess, and S. K. Burgess, "Infrared filter system for fluorescent lighting," US, 0252507, 2004.

[23] T. Zhu, K. Shanmugasundaram, F. Zhang et al., "Mist fabrication of light emitting diodes with colloidal nanocrystal quantum dots," Applied Physics Letters, vol. 92, no. 2, Article ID 023111, 3 pages, 2008.

[24] X. Peng and L. Qu, "Control of photoluminescence properties of CdSe nanocrystals in growth," Journal of the American Chemical Society, vol. 124, no. 9, pp. 2049-2055, 2002. 

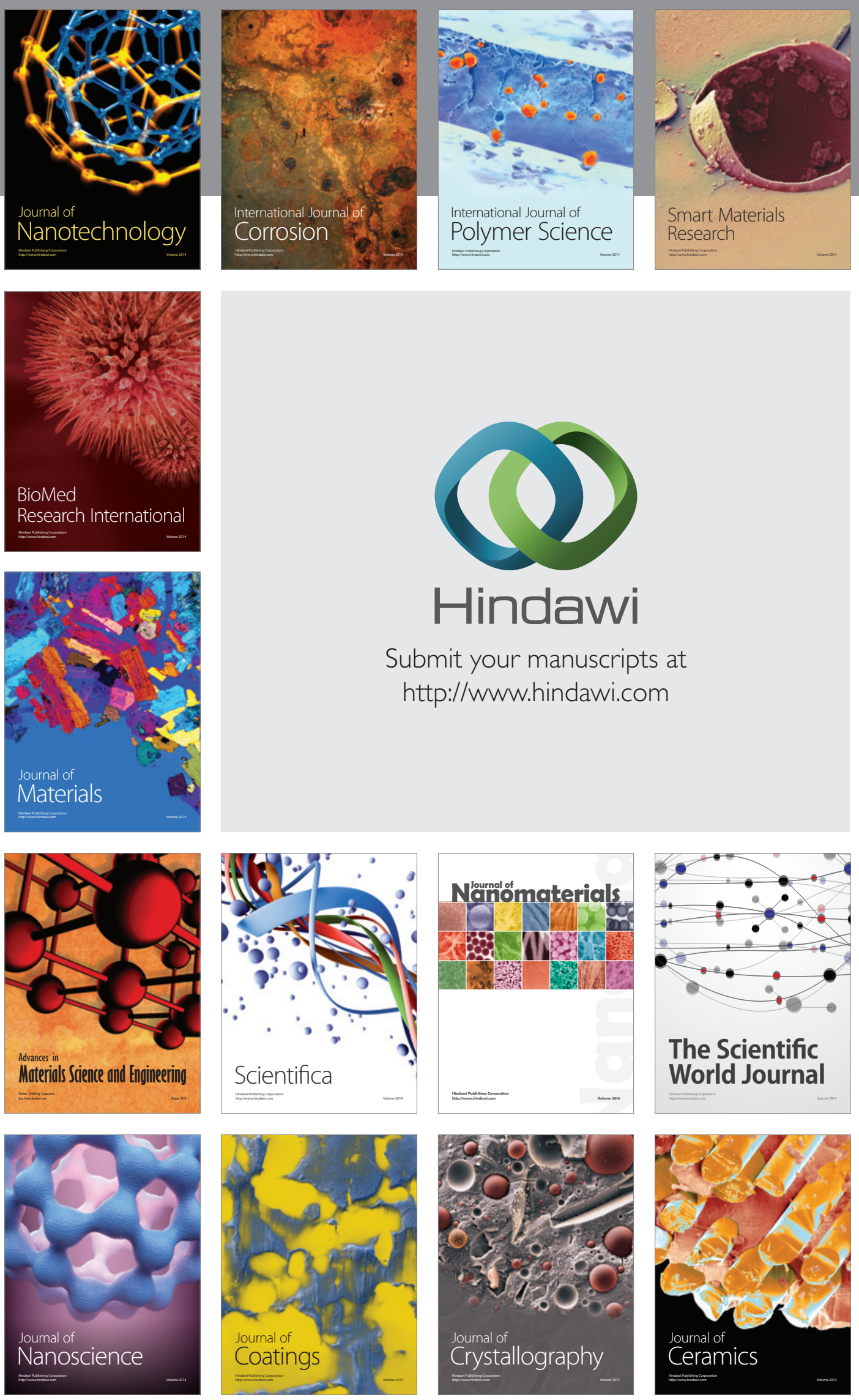

The Scientific World Journal

Submit your manuscripts at

http://www.hindawi.com

\section{World Journal}

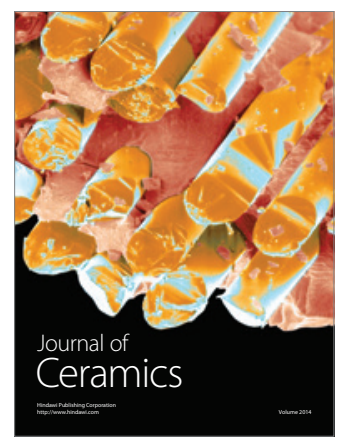

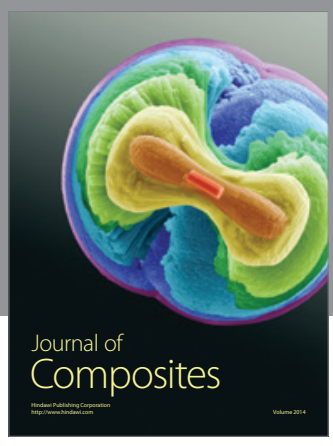
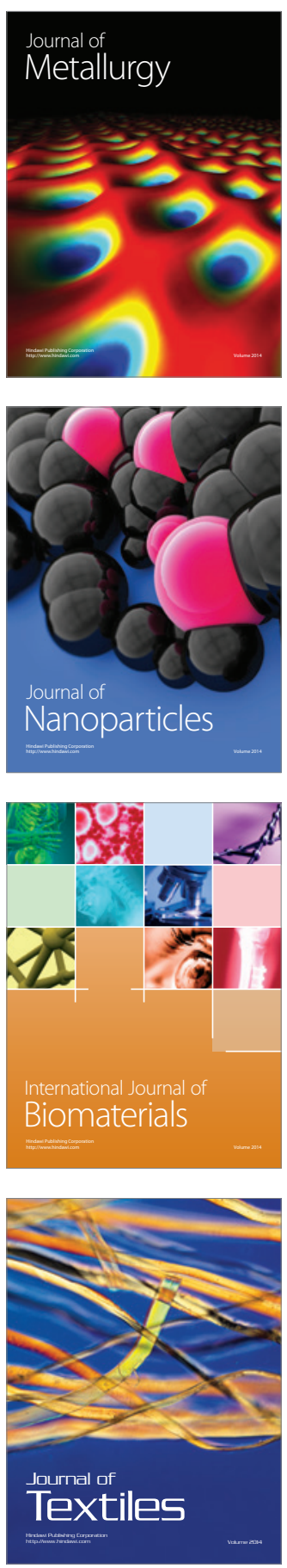